See discussions, stats, and author profiles for this publication at: https://www.researchgate.net/publication/329597843

\title{
Managing the Process of Servicing Hybrid Telecommunications Services. Quality Control and Interaction Procedure of Service Subsystems
}

Chapter · January 2019
DOI: $10.1007 / 978-3-030-03314-9+2$

\section{CITATIONS}

7

4 authors:

Mariia Skulysh

National Technical University of Ukraine Kiev Polytechnic Institute 38 PUBLICATIONS 62 CITATIONS

SEE PROFILE

Larysa S Globa

National Technical University of Ukraine Kiev Polytechnic Institute 149 PUBLICATIONS 185 CITATIONS

SEE PROFILE
READS

78

Oleksandr Ivanovich Romanov

National Technical University of Ukraine "Igor Sikorsky Kyiv Polytechnic Institute" 15 PUBLICATIONS 37 CITATIONS

SEE PROFILE

Iryna Husyeva

National Technical University of Ukraine Kiev Polytechnic Institute

1 PUBLICATION 7 CITATIONS

SEE PROFILE

Some of the authors of this publication are also working on these related projects:

Project Load management in the heterogeneous data centers of the operator View project 


\title{
Managing the process of servicing hybrid telecommunications services. Quality control and interaction procedure of service subsystems
}

\author{
Mariia A. Skulysh, Oleksandr I. Romanov, Larysa S. Globa and Iryna I. Husyeva
}

\author{
National Technical University of Ukraine \\ «Ihor Sikorsky Kyiv Polytechnic Institute» \\ Kyiv, Ukraine \\ mskulysh@gmail.com \\ a_i_romanov@gmail.com \\ lgloba@its.kpi.ua \\ iguseva@yahoo.com
}

\begin{abstract}
The principle of telecommunication system management is improved. Unlike the principle of software-defined networks, the functions of managing the subscriber service process, namely: subscriber search, the search for the physical elements involved in the transmission process, and the transfer of control to the corresponding physical elements, are transferred to the cloud. All subsystems of mobile communication will be managed from controllers located in the date center. The interaction between subsystems controllers for managing occurs only in the data center. It will reduce the number of service streams in telecommunications network. The procedure of interaction of the mobile communication control system and the virtualized environmental management system is proposed.
\end{abstract}

Keywords: NFV, VeCME, VBS, VeEPC, LTE, 5G, TC gibrid-service.

\section{Introduction}

The work of the telecommunications network is inextricably linked with computer systems. According to [1], a hybrid telecommunications service is a service that includes components of cloud and telecommunications services. A mobile network consists of a local area network, a radio access network and a provider core network. The advent of cloud computing has expanded the possibilities for servicing telecommunications systems.

Specifications [2] represents the main architectural solutions in which complex hardware solutions are replaced by different ways of virtualizing network sections. This allows configuring the network computing resources in a flexible way. To do this, it is necessary to create new methods of managing the quality of service that will take into account the features of the process in the telecommunication system and in the computing environment for servicing hybrid services.

The purpose of this work is to improve the quality of service of hybrid telecommunication services. To this end, it is proposed to use methods to control the formation of service request flows and to manage the allocation of resources. Realization of the set goal is achieved by solving the following tasks: 
1. Research of developments of the scientific community in the field of monitoring and ensuring the quality of service of hybrid services. Identification of processes regularities and features.

2. Development of a model of servicing hybrid services for a heterogeneous telecommunication environment.

3. Development of methods for ensuring the quality of service in access networks.

4. Development of methods for ensuring the quality of service in the local networks of the mobile operator and on their borders.

5. Development of a model and methods for the operation of the provider core network in a heterogeneous cloud infrastructure

6. Development of a functioning model for provider charging system.

To realize these tasks, it is necessary to take into account such factors as the annual growth of traffic volumes in an exponential progression; the need for differentiated services for a multiservice flow and different quality of service requirements; the need for constant monitoring of quality indicators and timely response to their decline.

Thus, the operator's monitoring system collects and processes a large amount of information about the quality of each service. It also monitors the telecommunications operator subsystems, the number of failures, etc.

For an adaptive response to the decline in quality of service today, such mechanisms are used:

- Monitoring the workload of the network;

- Monitoring of queue service quality in communication nodes;

- Managing of subscriber data flows;

- Managing of queues for differentiated servicing of multi-service flows

- Overload warning mechanisms;

- Methods of engineering the traffic for an equable distribution of resources.

\section{Organization of a heterogeneous telecommunications network}

According [3], all calculative functions that accompany transfer process are performed in data centers with cloud infrastructure. Virtualization of the base station will reduce the amount of energy consumed by the dynamic allocation of resources and load balancing. In addition to virtual base stations, radio access networks with cloud-based resources organization (Cloud-RAN) is required to create a resource base frequencies processing, which will combine different computing resources of centralized virtual environment. The specification offers virtualization of network functions for the router located on the border of provider local network. A router performs flow classification, routing management and providing firewall protection.

For the organization of virtual base stations and VeCPE it is necessary for data center to be close to base stations and to each output of the local network. So, the of the provider network represents a geographically distributed network of data centers with communication channels delivered primary information of mobile subscribers to each of them. The network requires conversion at the lowest level, so the signal requires recognition and decoding at higher levels of MAC, RLC, RRC, and PDCP. The specification is also propose provider core virtualization. 
Based on this, it can be assumed that most of the network processes are performed in datacenters, and the network is only a means of delivering information messages [4]. In the conditions of program-controlled routers distribution, there is network structure shown in Fig. 1.

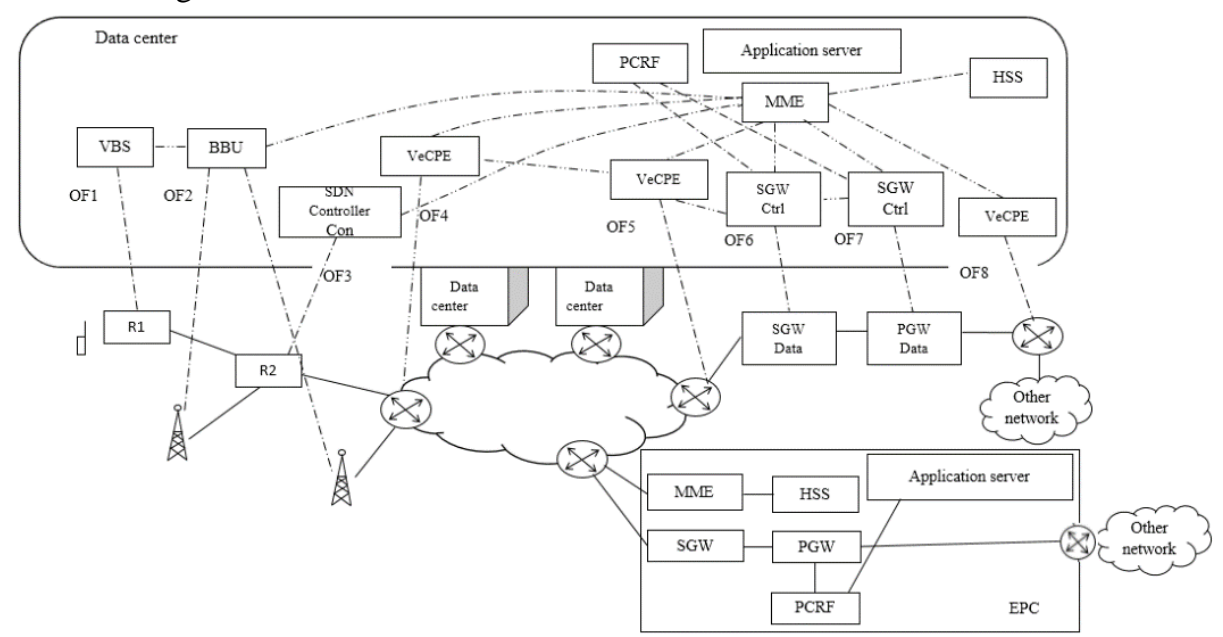

Fig.1. Provider core network structure using software-controlled routers

Fig. 1 shows how the mobile subscriber communicates with the R1 transponder, which converts the radio signal to optical, and then the signal reaches the R2 transponder managed by the SDN controller, which is also situated in the data center. After attaining the data center, the signal is processed by the virtual base station. Further, according to LTE technology, the flow is sent to the operator's core for further processing. The BBU subsystem is based on the technology of software-configurable networks/virtualization of network operation. This system supports either the work of virtual base stations or hybrid of 2G/3G/4G/Pre5G solutions.

Further direction of data channels is determined by servicing in the core. If the flow is directed to the provider internal network, it is immediately sent to the corresponding virtual base station in the data center for service, and then forwarded to the subscriber through the transponders R2 and R1. If the stream is to be sent outside the operator's local network, it is directed to the boundary virtual router, and then to external networks. This is the example of Next Generation Network

Thus, the data center combines a group of data centers that are connected to a single logical space for servicing virtualized network functions through a secure network. The quality of end users service is influenced by the organization of processes in such a heterogeneous data center based on the cloud computing concept.

According to the ITU-T Y.3500 recommendation, cloud computing is the paradigm network access providing to a scalable and flexible set of shared physical and virtual resources with administration based on on-demand self-service.

The structure of described data center in which the group of functional blocks shown in fig. 1 are servicing is shown in fig. 2. There is a transport network and connected data centers, forming a single virtualized space. 


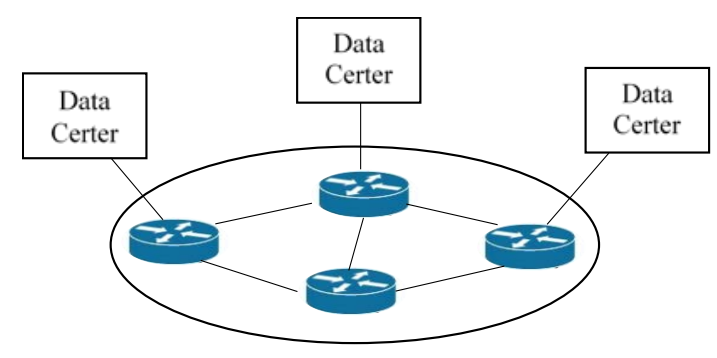

Fig. 2. The structure of the heterogeneous data center

Recommendation ITU-T Y.3511 defines this complex system of data center groups as multi-cloud computing. It is a paradigm for interaction between two or more providers of cloud services. Recommendation ITU-T Y.3520 presented the conceptual architecture of the multi-cloud and multi-platform cloud services management presented in fig. 3 [5]

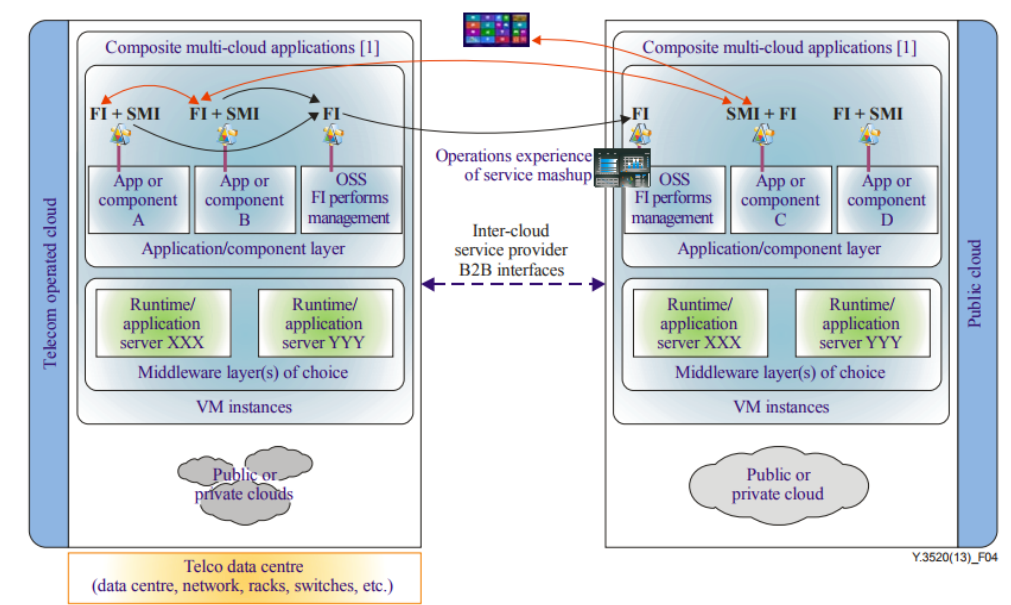

Fig. 3. Architectural vision for multi-cloud, multi-platform cloud management

During the work of provider data center, virtual BS system, the core subsystems and the virtual router are in a single logical space. In fig. 3 we can see that at the middleware level XXX Server is presented in every data center that participates in the inter-cloud computing infrastructure. The corresponding programs that activate the provider functional blocks are performed at the application and component level.

To ensure the work of mobile network using virtualization technology, it is necessary to provide a distributed structure of data centers, organized in a single virtual space. The structure should include deployed logical elements of the mobile service network, process management and flow allocation carried out by the orchestrator (fig. 4). 


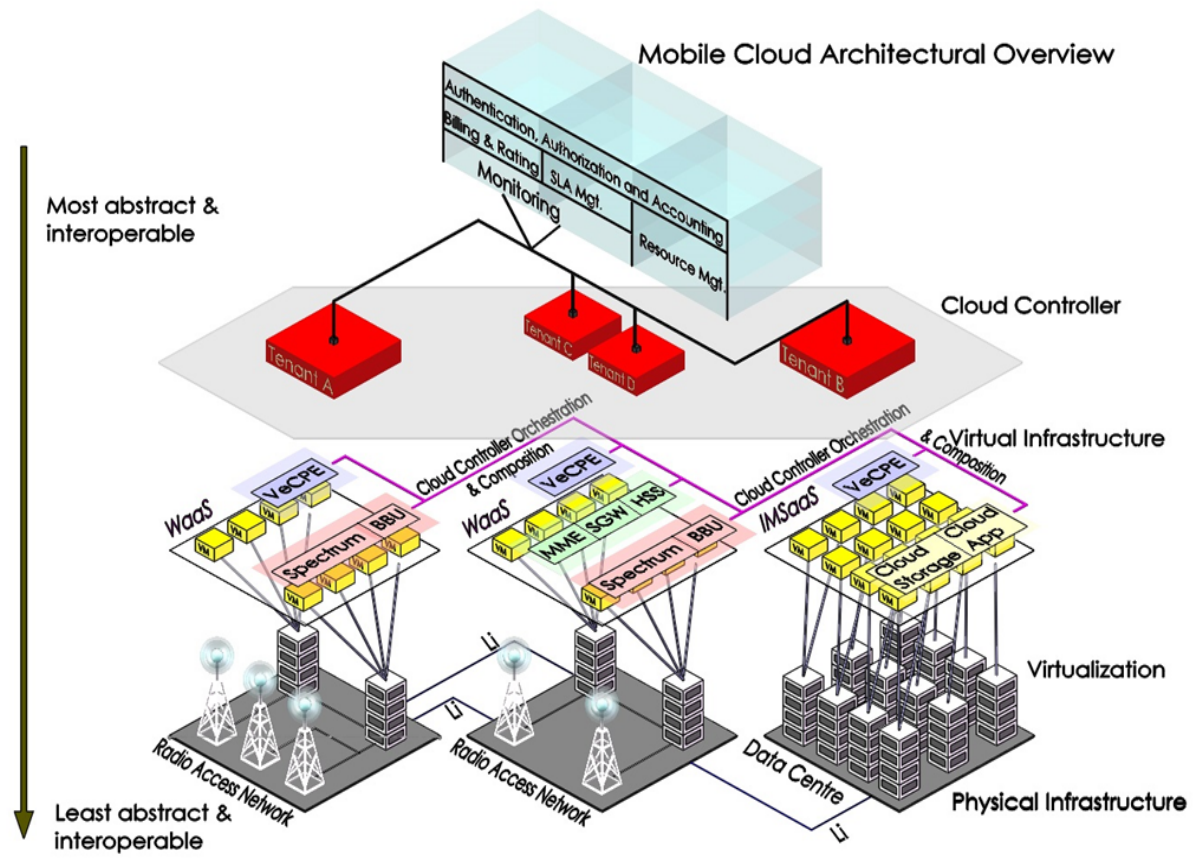

Fig. 4. Organization of service in New Generation Networks

According to the research, the effectiveness of computing processes organization in functional units affects the efficiency of end-users servicing of a mobile operator. The data processing center in this architectural solution is a complex organizational and technical set of computing and telecommunication systems that ensures the smooth operation of the NFV infrastructure. The effectiveness of its operation depends on the choice of physical data centers that will become part of the distributed center structure; the location of network functions in the infrastructure; the organization of flows between virtualized objects and the allocation of resources for their servicing.

\section{The principle of flow service with the resource virtualization in public telephone network}

Controllers located in the data center guide all subsystems of mobile communication. The interaction between controllers of subsystems for the purpose of control occurs only in the middle of the date center. The functions of managing the service process, namely: searching for the subscriber, searching for the physical elements involved in the transmission process, and passing the guidance on the corresponding physical elements, are transferred to the cloud.

The subscriber device for connection organization interacts with the base station controller located at the data center. According to the protocols, subsystem controllers interact at the level of the data center, sending the final hardware solutions to the physical equipment to start the data transmission process (Fig. 5). 


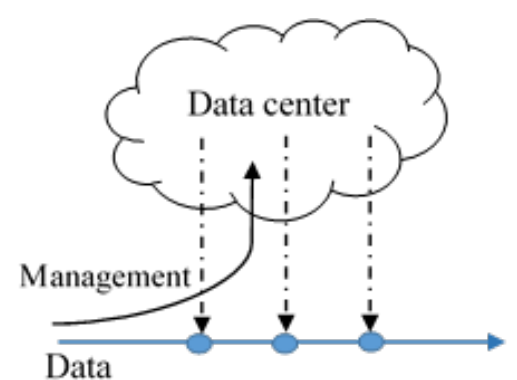

Fig. 5. The principle of flow service with the resource virtualization in public telephone network

There are two principles of virtualization of network resources. The first principle redirects through the cloud resources only control flows. The second principle is to use cloud-based data centers to process both network and information flows. In this paper, the first principle is considered. According to it, virtualization of network functions allows separating the control system of the mobile network nodes from the data transmission system. In fig. 6 you can see an example of separating the functions of the core network. The main functions of the core subsystem were analyzed, and the functions associated with the control and data transfer were selected. Data transfer functions are solved in a virtualized environment deployed on the basis of data centers group [6].

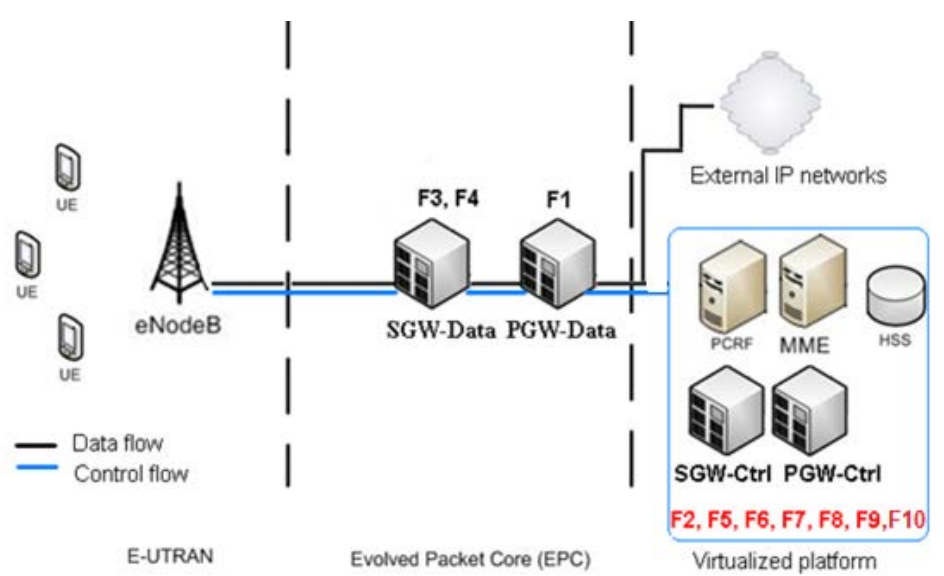

Fig. 6 Distribution of network core functions between physical and virtual devices

F1 - packet filtering by users and legitimate interception of traffic;

F2 - IP pool distribution functions for UE and PCEF Functionality;

F3 - basic routing and interception of packet traffic;

F4 - the function of an anchor point (traffic aggregation point) for a handover between the NodeBs within one access network in the base station service area according to a set of rules and instructions;

F5 - processing of BBERF functionality;

F6 - Traffic Detection Function;

F7 - User Data Repository (UDR);

F8 - Application Function (AF); 
F9 - Online Charging System (OCS).

Fig. 7 shows the processes of network subsystems interaction with the separation of control functions and data transmission with virtualization in the provision of data transfer functions. In fact, each arrow on this scheme is a service request in this virtual (or physical) node. The number of requests per time unit is the load intensity on given service node.

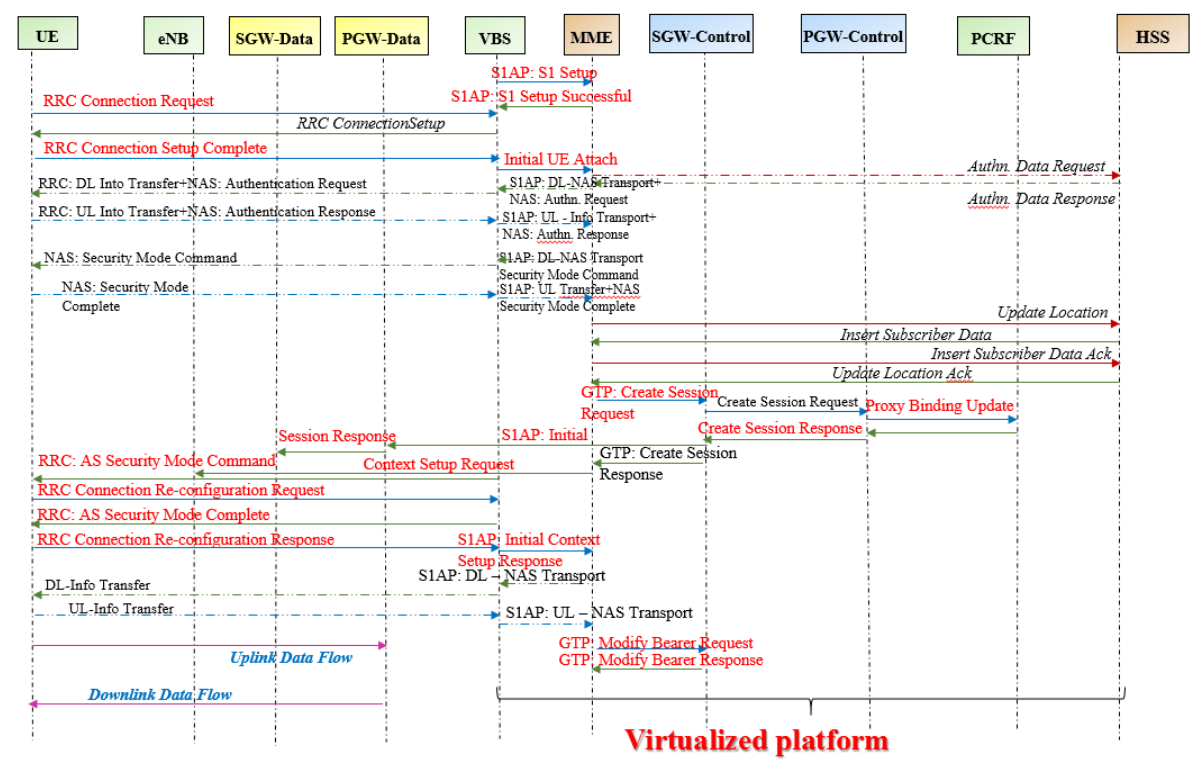

Fig. 7. Procedure of subsystems interaction during subscriber's service

Network structure and user service quality control take place in the nodes. Traditionally, the subsystems of LTE network perform a set of functions, in accordance with standards and specifications. The paper proposes to divide subsystem management functions and functions that are associated with the data transfer process directly to the LTE network. The feature is the expansion of subsystems functionality, compared with the networks of previous generations. More than half of the subsystem functions are connected not with the service process, but with the management of the communication system. Service quality control occurs in the subsystems eNodeB, SGSN, PCRF (fig. 8). Delay control in virtualized network nodes, where service intensities depend on computing resources requires PCRF modification. 


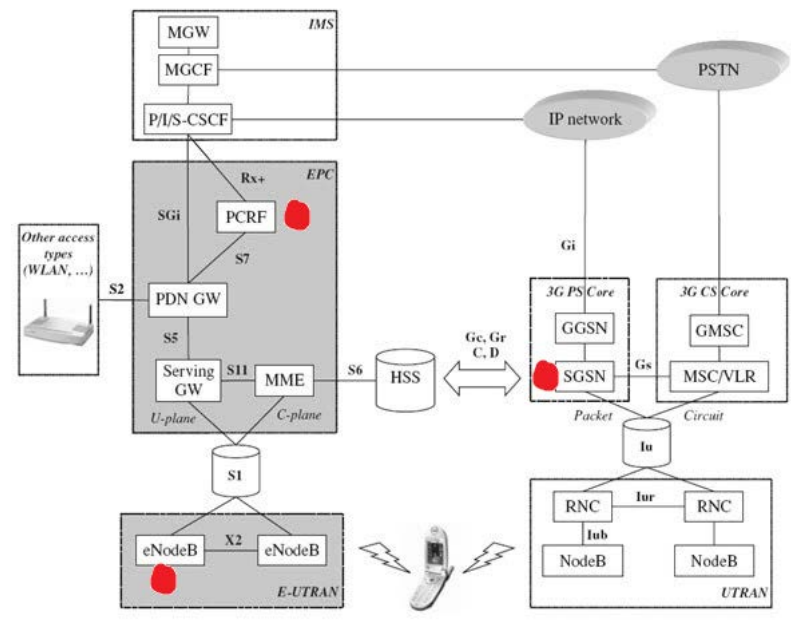

Fig. 8. General architecture of the standard LTE network

The efficiency of hybrid telecommunication services is estimated by quantitative indicators of service quality:

- $\quad t_{d}$ - time delay in the maintenance of the hybrid telecommunication management service $\left(t_{\text {data }}-t_{\text {start }}\right)$, where $t_{\text {start }}$ is the moment of request by the subscriber for permission to transmit data information flows, $t_{\text {data }}$ is the moment when the subscriber begins to transmit information streams;

- $\quad P-$ the probability of refusal in service.

$$
P=\prod_{i=1}^{N} P_{i}
$$

where $P_{i}$ is failure probability in virtualized service node for one of the requests types to the subsystem of the heterogeneous telecommunication environment.

\section{Procedure of guaranteeing the adjusted quality of service}

The principle of dynamic quality control is as follows: the delay value in maintaining the application for connection (disconnection, recovery) is compared with the service quality policy of the subscriber. If the metric does not match, then the quality metrics in virtual nodes and VLANs are consistently compared with the thresholds of the corresponding policies stored in the PCRF subsystem. This principle analyzes the following quantitative indicators of the effective system operation, such as: the time of service flow request delay in the virtual node and the probability of queries loss in the service node. Service node is a virtual machine that performs the functions the network node managing.

After discovering the reason of service efficiency indicators problem, then appropriate measures are taken. If there is a problem in the time of transmission between service nodes, then it is recommended to reconfigure the system, namely to change the location of virtual nodes in physical nodes of the heterogeneous data center structure. If the problem is identified in one service nodes, then it is recommended to 
increase the number of service resources. If there is a decrease in service quality rates in a group of linked interface nodes, for example, which form a single core of the EPC network, then it is recommended to limit the flow of applications sent to service the corresponding core. For this purpose it is recommended to calculate the intensity of the load on the group of nodes. The algorithm of the procedure is shown in fig. 9

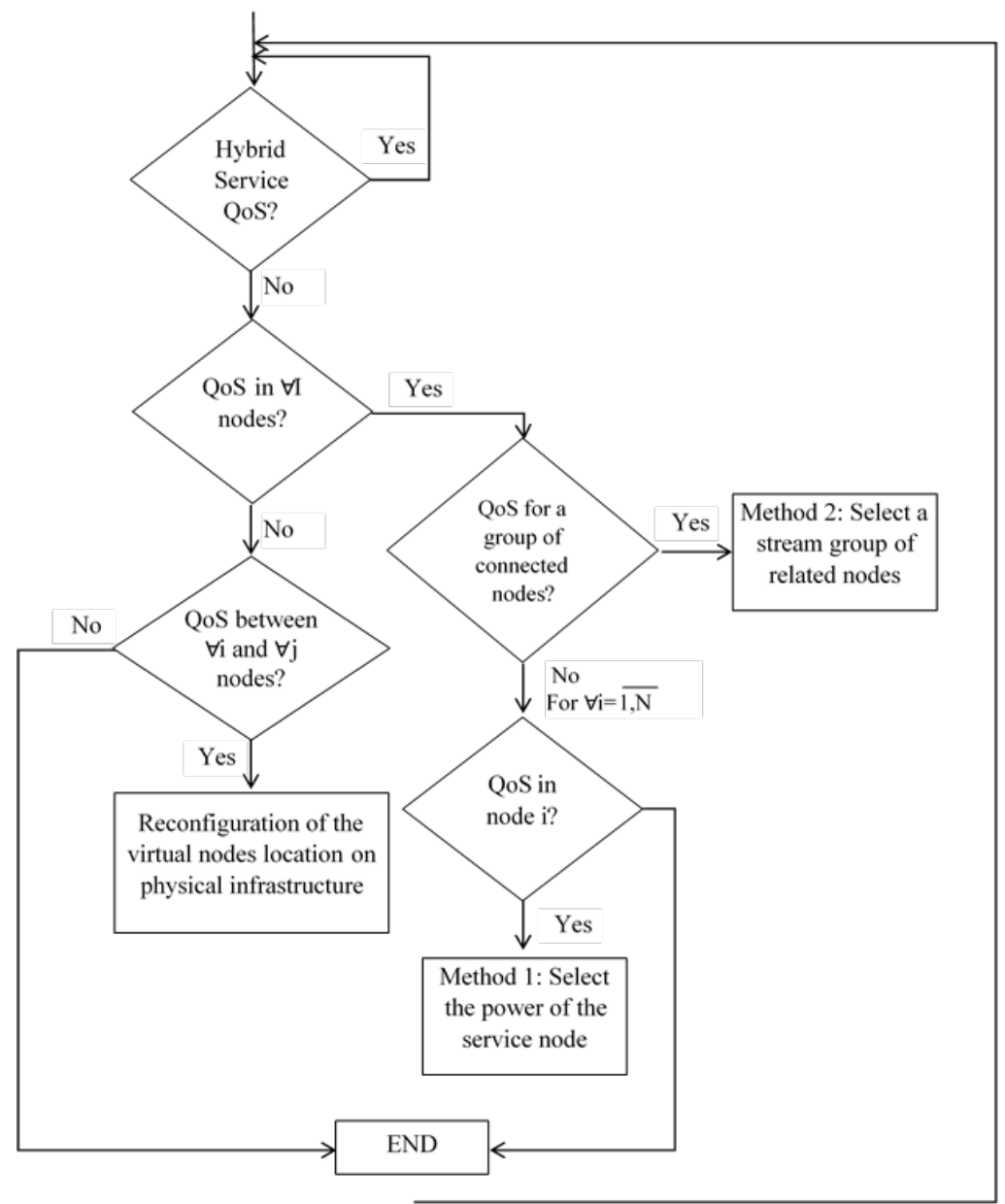

Fig. 9. Procedure for guaranteeing the preset quality of service

To implement the principle of dynamic quality control, a modification of the PCRF system subsystems is required. The "Single Policy Storage" subsystem is expanding, and the following policies regarding quality management service flow rates are added:

1. The allowable delay time for an application service flow in a virtual host.

2. Permissible loss of requests in the virtual node

3. Permissible time for serving requests in groups of virtual nodes that provide a given service.

4. Permissible delays in transmission between service nodes

5. The value of the admissible delivery delays of the guiding influence on network nodes. 
An expanded subsystem is shown in Fig. 10

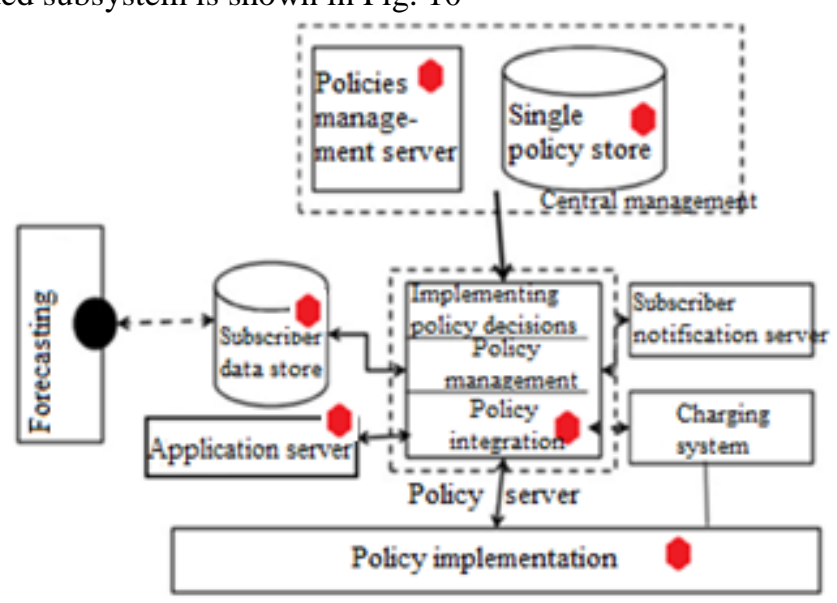

Fig. 10. PCRF subsystem modification

- The "Policy Management" subsystem creates a set of requirements for implementing a set of policies in relation to different flows of management.

- The "Policy Server" subsystem detects a problem of inconsistency of the current quality metrics with the declared subscription service policies.

- In the "Application Server" subsystem, program modules in which calculations are performed according to the proposed methods are implemented. The source data for the methods is the statistics obtained from the monitoring system and policy data that is provided to respective subscribers.

- The "Subscriber Data Store" subsystem is supplemented by information about virtual nodes, or a separate virtual network maintenance statistics database is created. This database collects information about service requests flows; the statistics of the relative dependence of the service intensity on service resources for each type of request.

The principle of dynamic quality control requires new procedures: it is necessary to arrange the interaction of mobile communication management system with virtualized resources management system (Fig. 11).

The quality control of management procedures implementation is evaluated at the level of User Equipment:

The User Equipment records the time delay in execution of service procedures, namely the time from the moment of connection initialization to the moment of data transmission beginning, and transfers to the subsystem of PCRF.

The PCRF receives this information from the subscriber and analyzes the policy server; in the policy implementation sub-system it compares the received data to the correspondence of chosen subscriber policy that is stored in the "Subscriber data store". 


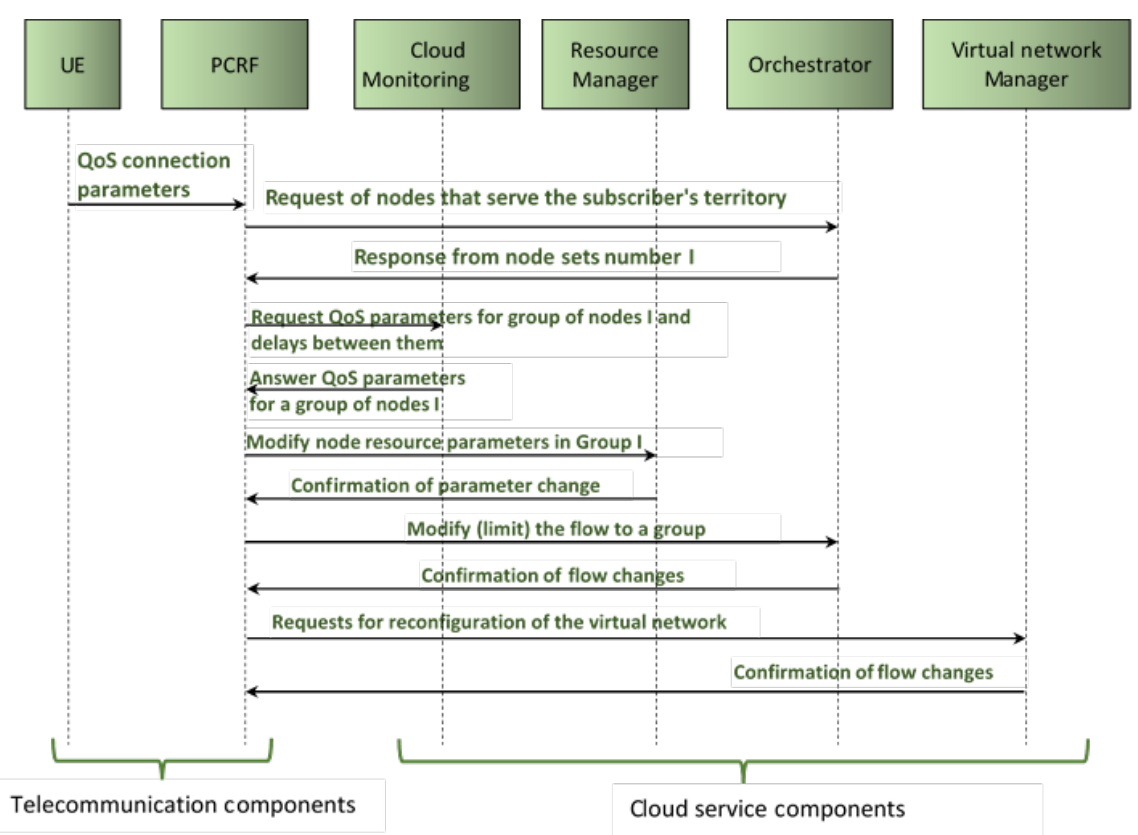

Fig. 11 Interaction of the mobile communication control system and the virtualized environment management system

If the delay values are not in accordance with the policy, PCRF requests the "Orchestrator" subsystem to identify the group of nodes $i$ that serve the subscriber.

Orchestrator sends the numbers of nodes serving the subscriber, located in a given area. PCRF sends request to "Cloud Monitoring" for information on the delay and loss parameters in the nodes $i$, and information on the delay between nodes services. The Cloud Monitoring collects information regarding the latency and loss performance of hybrid services that are served on the nodes of the virtual network. The data about the service node group is transferred to the PCRF, where the principle of dynamic quality control of the service of hybrid services is realized. According to the management decisions, the PCRF subsystem sends inquiries:

- for reconfiguration of the virtual network, to the "Virtual Network Manager";

- to reconfigure resources to "Resource Manager";

- to change flows of service to "Orchestrator" streams over a virtual network.

When implementing the principle of dynamic quality control, most subsystems of the PCRF system are involved.

\section{$5 \quad$ Conclusions}

An approach to managing a heterogeneous telecommunication environment for increasing the efficiency of the service process of hybrid telecommunication services in new generation systems is proposed. A unified solution for telecommunication systems, where the maintenance of hybrid telecommunication services is carried out with the use of software is proposed. This approach allows to avoid reducing the quality of service during dash of overload and to maintain quality of service indicators at a 
given level, subject to compliance the resource utilization rate within the specified limits.

The modification of PCRF subsystems and new procedures for organizing the interaction of the mobile telecommunication network subsystems and the virtualized environmental management subsystems is proposed. It provides a process for monitoring the quality of service of hybrid telecommunication streams in the telecommunication environment, which allow providing the quality of service control and planning the amount of service resources for the efficient operation of heterogeneous telecommunication environment.

\section{References}

1. ITU-T Recommendation M.3371 of October 2016.

2. ETSI GS NFV 001 v.1.1.1 (10/2013).

3. ETSI GS NFV 001 v.1.1.1 (10/2013).

4. Skulysh M. Romonov O. The structure of a mobile provider network with network functions virtualization // TCSET 2018 : 14-th International conference on Advanced Trends in Radioelectronics, Telecommunications and Computer Engineering, 20-24 February 2018 : conference proceedings. - Lviv-Slavske, 2018. - P. 1032 - 1034.

5. J. ITU-T Y.3520 Telecommunication standardization sector of ITU (06/2013) / Series Y: Global information infrastructure, internet protocol aspects and next-generation networks.

6. Skulysh M., Klimovych O. Approach to virtualization of Evolved Packet Core Network Functions //Experience of Designing and Application of CAD Systems in Microelectronics (CADSM), 2015 13th International Conference The. - IEEE, 2015. - C. 193-195.

7. Larysa G. et al. Managing of incoming stream applications in online charging system //Telecommunications (BIHTEL), 2014 X International Symposium on. - IEEE, 2014. - C. $1-6$.

8. Mariia Skulysh. The method of resources involvement scheduling based on the long-term statistics ensuring quality and performance parameters //Radio Electronics \& Info Communications (UkrMiCo), 2017 International Conference.

9. Globa L. Method for resource allocation of virtualized network functions in hybrid environment / L. Globa, M. Skulysh, S. Sulima // 2016 IEEE International Black Sea Conference on Communications and Networking Pages: 1 - 5, DOI: 10.1109/BlackSeaCom.2016.7901546.

10. Olena Semenova, Andriy Semenov, Oleksandr Voznyak, Dmytro Mostoviy, and Igor Dudatyev, "The fuzzy-controller for WiMAX networks," Proceedings of the International Siberian Conference on Control and Communications (SIBCON), 21-23 May 2015, Omsk, Russia, pp. 1-4. DOI: 10.1109/SIBCON.2015.7147214. 